\title{
An architectonic type principle in the development of laminar patterns of cortico-cortical connections
}

\author{
Sarah F. Beul ${ }^{1} \cdot$ Alexandros Goulas $^{1} \cdot$ Claus C. Hilgetag ${ }^{1,2}$ (1)
}

Received: 25 October 2019 / Accepted: 12 January 2021 / Published online: 9 February 2021

(c) The Author(s) 2021

\begin{abstract}
Structural connections between cortical areas form an intricate network with a high degree of specificity. Many aspects of this complex network organization in the adult mammalian cortex are captured by an architectonic type principle, which relates structural connections to the architectonic differentiation of brain regions. In particular, the laminar patterns of projection origins are a prominent feature of structural connections that varies in a graded manner with the relative architectonic differentiation of connected areas in the adult brain. Here we show that the architectonic type principle is already apparent for the laminar origins of cortico-cortical projections in the immature cortex of the macaque monkey. We find that prenatal and neonatal laminar patterns correlate with cortical architectonic differentiation, and that the relation of laminar patterns to architectonic differences between connected areas is not substantially altered by the complete loss of visual input. Moreover, we find that the degree of change in laminar patterns that projections undergo during development varies in proportion to the relative architectonic differentiation of the connected areas. Hence, it appears that initial biases in laminar projection patterns become progressively strengthened by later developmental processes. These findings suggest that early neurogenetic processes during the formation of the brain are sufficient to establish the characteristic laminar projection patterns. This conclusion is in line with previously suggested mechanistic explanations underlying the emergence of the architectonic type principle and provides further constraints for exploring the fundamental factors that shape structural connectivity in the mammalian brain.
\end{abstract}

Keywords Cortical architecture $\cdot$ Development $\cdot$ Structural connectivity $\cdot$ Tract-tracing

\section{Introduction}

Brain regions are linked by a complex, characteristically organized network of structural connections. One principle that has been shown to capture reliably multiple topological features of cortico-cortical connectivity in the adult mammalian brain is the structural model (Barbas 1986, 2015; Barbas and Rempel-Clower 1997; García-Cabezas et al. 2019), also called architectonic type principle (Hilgetag et al. 2019). This model relates fundamental features of mesoscopic

Alexandros Goulas, Claus C. Hilgetag have are shared senior authors.

Claus C. Hilgetag

c.hilgetag@uke.de

1 University Medical Center Hamburg-Eppendorf, Martinistr. 52, 20246 Hamburg, Germany

2 Department of Health Sciences, Boston University, 635 Commonwealth Avenue, Boston, MA 02215, USA structural connections, such as their existence or layerspecific patterns of projection origins and terminations, to the relative architectonic differentiation of the brain. The architectonic type principle accounts for data observed in a number of mammalian species, such as the rhesus macaque (Medalla and Barbas 2006; Medalla et al. 2007), the cat (Hilgetag and Grant 2010) or the mouse (Goulas et al. 2017), connections within as well as between the cortical hemispheres (Barbas et al. 2005; Goulas et al. 2017), connections towards the primate frontal cortex (Barbas 1986; Barbas and Rempel-Clower 1997; Rempel-Clower and Barbas 2000; Ghashghaei et al. 2007), within the primate and cat visual system (Hilgetag and Grant 2010; Hilgetag et al. 2016) and connections across the entire cortex of the macaque (Beul et al. 2017; Beul and Hilgetag 2019) and the cat (Beul et al. 2015). It has been suggested that such a widely applicable principle may emerge from spatio-temporal interactions of neural populations during ontogeny, without the essential need of sensory input or other major external influences (Barbas 1986, 2015; Barbas and García-Cabezas 2016; 
Hilgetag et al. 2016). The experimental study of the formation of structural connections, which happens concurrently with the formation of the brain itself, is an onerous endeavour. Consequently, the first support of the suggested mechanistic explanation for the emergence of the architectonic type principle has come from in silico models, where simulation results have shown that simple interactions between the time and place of neurogenesis can result in structural networks that capture many of the relationships observed in empirical mammalian cortico-cortical connections (Beul et al. 2018; Goulas et al. 2019). These simulation experiments have explored how the existence of connections (i.e., whether brain areas are connected or not) and the strength of projections can be shaped. Naturally, further features of connectivity, beyond the basic existence of connections, are of interest. One of these features is presented by the characteristic layer-specific patterns of cortical projection origins and terminations, which are strikingly regular (Rockland and Pandya 1979; Felleman and Van Essen 1991; Hilgetag et al. 2000) and well captured by the architectonic type principle (Barbas 1986; Barbas and Rempel-Clower 1997; RempelClower and Barbas 2000; Hilgetag et al. 2016). In terms of projection origins, the patterns can be broadly categorized into projections of mostly supragranular (upper layer) origin, which are typical of projections from external to more internal cortical areas. By contrast, the projections of approximately balanced upper and deep layer origins form connections between similar areas, while the projections of mostly infragranular (deep layer) origin contribute to projections from more internal to external cortical areas as well as to subcortical projections (reviewed in García-Cabezas et al. 2019; Hilgetag et al. 2019). Layer-specific projection patterns represent a connectional feature that is fundamental to advanced theories of relations between brain structure and function (Friston 2010; Feldman Barrett and Simmons 2015), but how these laminar patterns are shaped during ontogeny is presently not fully clear. Since the architectonic type principle is the most central predictor of laminar projection patterns in multiple species as documented so far (cf. Barbas 2015; Hilgetag et al. 2019), there are two prominent questions about the origin of this relationship between architectonic differentiation and laminar patterns. First, it is not clear if the relation of architectonic differentiation and laminar origin of connections applies only to the adult state of the cerebral cortex. It was shown that the laminar origin of connections is not uniform, but already biased across areas early in development (Barone et al. 1995). Hence, one may wonder if the architectonic type principle reflects graded differences of the laminar origin of connections already in prenatal and neonatal states of the connectivity, or if the early laminar origin patterns of areas undergo drastic reconfigurations which alter the initial bias and thereby eventually give rise to the architectonic type principle in the adult animal.
In addition to the extent to which a biased distribution of laminar origins constitutes a pre-configuration of the adult state, a second question concerns the mechanisms that effect the refinement of laminar projection patterns. These could be intrinsic factors, such as apoptosis, or extrinsic factors, such as synaptic activity resulting from sensory input. Enucleation experiments allow inferring the influence of the visual input on the formation of cortical areas and connections, e.g., Karlen and Krubitzer (2009), and are thus helpful in deciphering the influence of external stimuli on the formation of connectional features.

Making use of tract-tracing data detailing laminar patterns of projection origins obtained in the immature macaque cortex (Kennedy et al. 1989; Batardière et al. 2002; Magrou et al. 2018), we here investigate the extent to which the architectonic type principle applies to the connectional data from early development and enucleated animals, presenting findings which indicate that the processes very early during ontogenesis are sufficient to establish laminar projection patterns that are consistent with the architectonic type principle.

\section{Methods and results}

To assess whether the laminar patterns of projection origins were correlated with relative architectonic differentiation of connected areas in the immature cortex of the macaque monkey, we combined five different resources providing measures of laminar projection patterns and architectonic differentiation.

\section{Projection data}

Measures of laminar projection patterns in the developing and adult macaque cortex were taken from previously published reports (Kennedy et al. 1989; Batardière et al. 2002; Chaudhuri et al. 2015; Magrou et al. 2018). Briefly, Kennedy et al. (1989) injected retrograde tracers in the neonate and adult striate cortex (area V1) of cynomolgus monkeys (Macaca irus). They evaluated labelled projection neurons in the posterior bank of the lunate sulcus (area V2), on the prelunate gyrus (area V4), and in the posterior bank and fundus of the superior temporal sulcus (STS, which we interpreted to correspond to areas FST, PGa, and STPi in the M132 parcellation of Markov et al. (2014); alternatively, we considered that STS may be equated to area V5/MT, or areas V5/MT and FST). For each observed projection, they determined the fraction of labelled neurons that originated in supragranular layers $\left(N_{\mathrm{SG}} \%\right)$. Batardière et al. (2002) followed a similar approach, injecting a retrograde tracer in area $\mathrm{V} 4$ of macaque monkeys (Macaca fascicularis) at different fetal stages (embryonic day 112 to embryonic day 140 ) and in adult monkeys. They evaluated labelled projection neurons across ten brain areas and also determined the 
proportional contribution of supragranular neurons $\left(N_{\mathrm{SG}} \%\right)$ to each projection (Batardière et al. (2002), their Fig. 7a).

Magrou et al. (2018) performed bilateral enucleation (removal of the eyes) in macaque monkey (Macaca fascicularis) fetuses between embryonic days 58 and 73. Retrograde tracers were injected into areas V2 and V4 postnatally, at postnatal day 16 and postnatal month 10 , respectively. Labelled projection neurons were evaluated across 18 and 16 brain areas, respectively, and the fraction of labelled projection neurons located in supragranular layers $\left(N_{\mathrm{SG}} \%\right)$ was determined. We compared the contribution from supragranular neurons in enucleated monkeys to $N_{\mathrm{SG}} \%$-values from intact adult macaque monkeys reported by Chaudhuri et al. (2015).

All $N_{\mathrm{SG}} \%$-values that we considered in our analyses are summarized in Online Resource 1.

\section{Measures of architectonic differentiation}

We considered two measures of architectonic differentiation of cortical areas, specifically architectonic type and neuron density. Architectonic type is an ordinal measure of differentiation assigned by experts based on a cortical area's overall appearance in different types of tissue stains, while neuron density is measured stereologically and has been shown to be a very distinctive marker of individual cortical areas (Dombrowski et al. 2001; Beul and Hilgetag 2019). Both measures have been published previously for the cortical areas considered here (Hilgetag et al. 2016) and are strongly correlated with each other (in this sample of areas, Spearman rank-correlation coefficient $\rho=0.96, p=3.9 \mathrm{e}-8$ ). Thus, in the present analysis, we used the same cortical type classification of areas as employed in Hilgetag et al (2016). The procedure that was used for categorizing cortical types in the Macaque, involving a combination of qualitative and quantitative criteria, is also described in detail in Hilgetag et al (2016). Architectonic type was available for all considered areas, and neuron density was available for all four areas considered by Kennedy et al. (1989), for 10 of the 11 areas considered by Batardière et al. (2002), as well as for 14 of the 20 areas considered by Magrou et al. (2018). We report results for both measures to present a comprehensive set of observations that is more robust against possible shortcomings of a particular measure.

\section{Immature projection patterns correlate with adult differentiation measures}

When immature (i.e., prenatal and neonatal) laminar patterns of projection origins are compared to their eventual adult composition, a clear correspondence can be observed, such that the bias in laminar projection origins that exists in the immature cortex largely persists in the adult cortex (Fig. 1a,
Online Resource 2). Consistent with this observation, immature patterns of laminar origins are strongly correlated with the difference in architectonic differentiation between the connected areas (Fig. 1b, c, Online Resource 2). For comparison, we also show the relation between adult $N_{\mathrm{SG}} \%$-values and difference in architectonic differentiation in these panels. Note that the slope of the regression lines becomes steeper for adult laminar patterns compared to immature patterns, indicating that an initial asymmetry in laminar contributions sharpens with maturation. The relation between immature and adult $N_{\mathrm{SG}} \%$-values becomes even clearer in Fig. 1d, e (also see Online Resource 2), which shows that the amount of remodelling that a projection undergoes from the immature to the adult state is also correlated with the connected areas' relative architectonic differentiation. This implies that later processes serve to refine a projection's laminar origins further towards a laminar bias that was already present from the outset. (For alternative results, in which the projection data for region STS were matched to area V5/MT, or areas V5/MT and FST, respectively, see Online Resource 3).

\section{Loss of visual input does not substantially alter the gradient of projection patterns}

The supragranular contribution to projections in enucleated infant monkeys is strongly correlated with the respective supragranular contribution in intact adult monkeys, especially if connections from highly affected area V1 (cf. detailed descriptions in Magrou et al. (2018) are excluded (Fig. 2A, Online Resource 4). However, there is a tendency towards higher supragranular contributions in the enucleated infants (as most data points are above the bisecting line), especially for projections to area V2. Indeed, a permutation test shows the median change in $N_{\mathrm{SG}}$ \% (i.e., enucleated $N_{\mathrm{SG}} \%$-intact $N_{\mathrm{SG}} \%$ ) to be larger for injections in V2 than in V4 ( $p=0.01,10^{4}$ permutations). Since the tracer was injected at different ages for projections to V2 and V4, the higher supragranular contribution could be explained by differences in maturation: V2 was injected earlier (at postnatal day 16) than V4 (at postnatal month 10), which may have caused the $N_{\mathrm{SG}} \%$ values of projections targeting $\mathrm{V} 4$ to be more similar to the intact adult $N_{\mathrm{SG}} \%$ values. This hypothesis is in line with the generally higher $N_{\mathrm{SG}} \%$ values observed for the prenatal and neonate injections reported by Batardière et al. (2002) and Kennedy et al. (1989). In principle, a comparison with neonatal projection patterns in intact monkeys would have been preferable to a comparison to adult patterns, but these data are not available for the projections between areas reported by Magrou et al. (2018). As it is, it might be argued that the projection patterns after enucleation are even less affected than it appears here, since the laminar patterns would likely undergo further postnatal changes, similar to the change already observed 


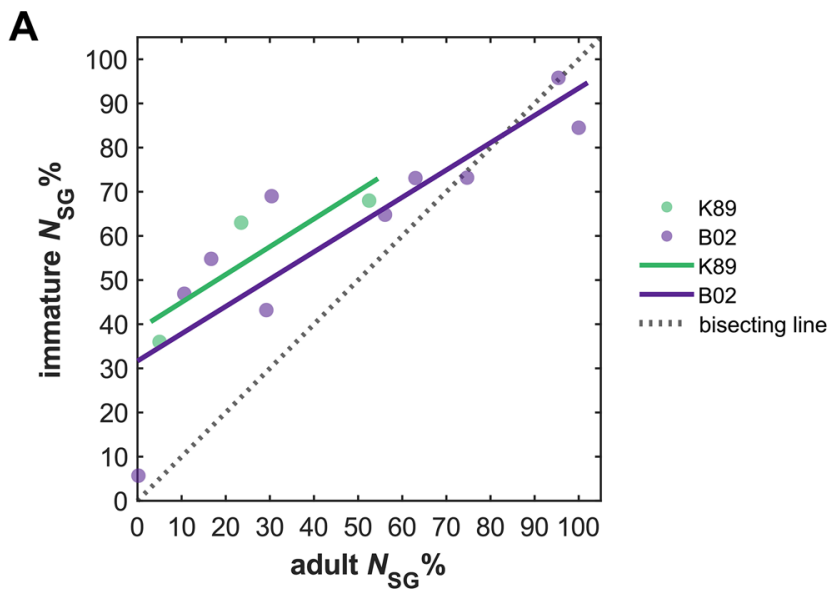

B

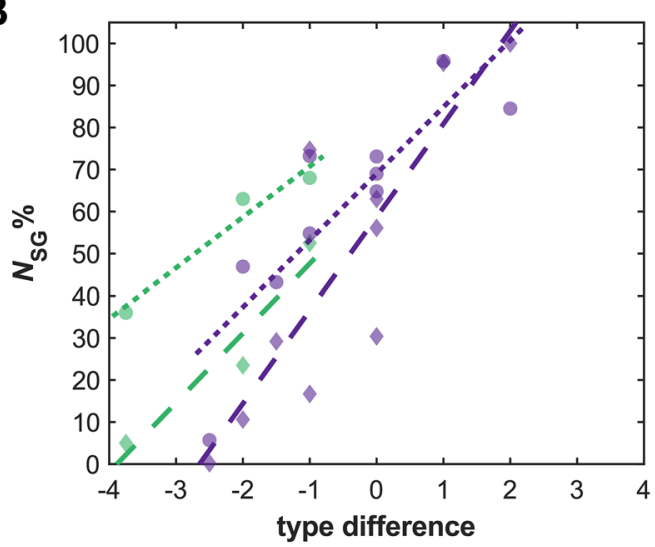

D

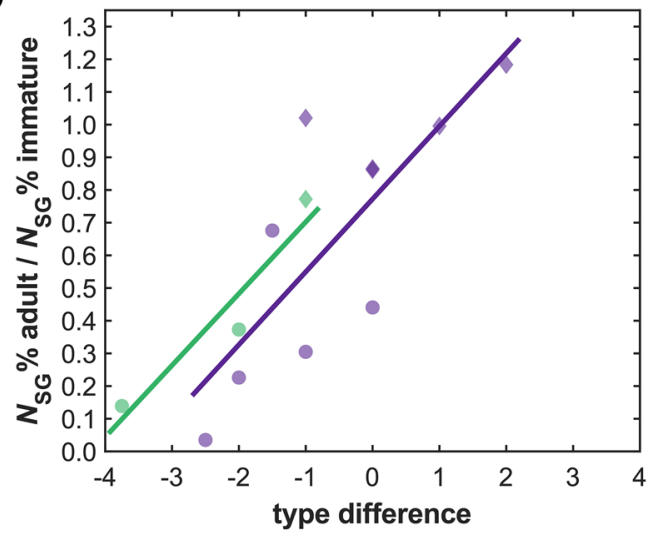

C

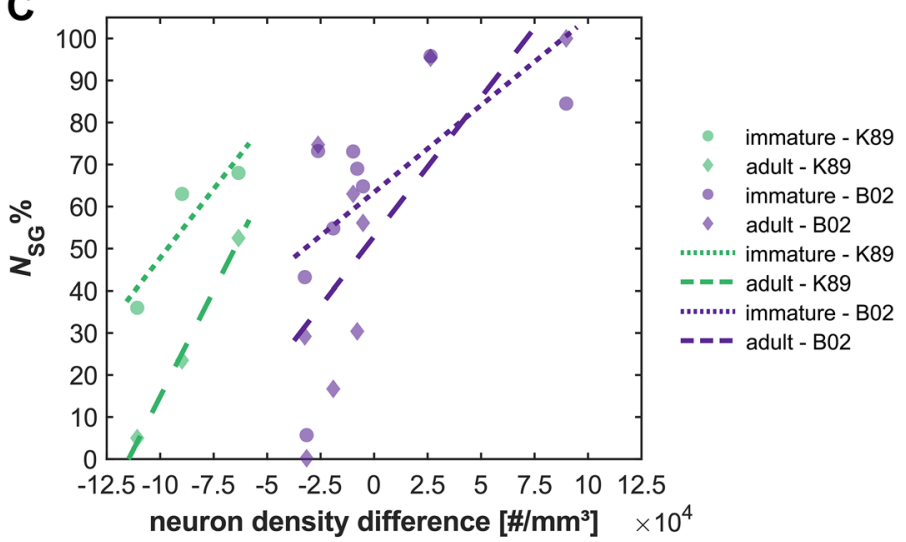

E

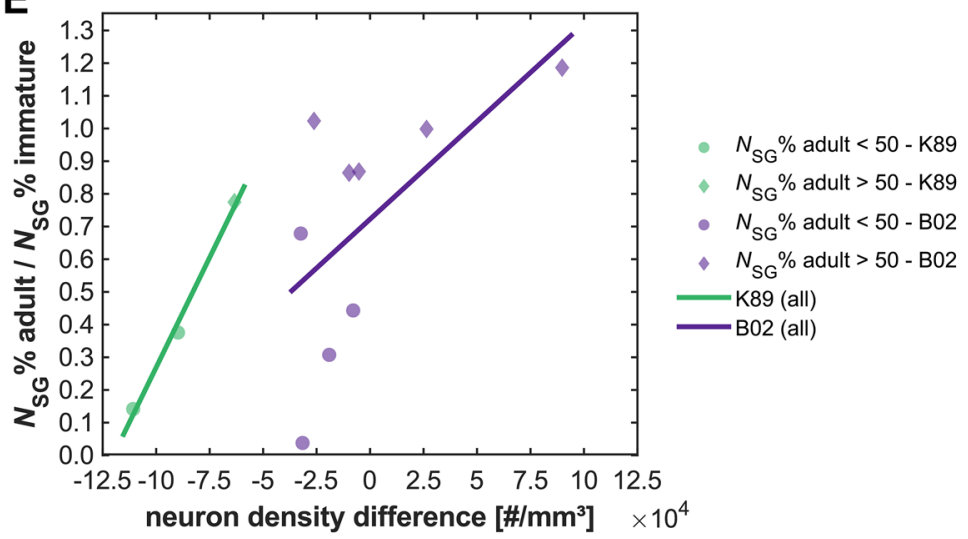

Fig. 1 Laminar projection patterns in immature cortex. Relative contribution of supragranular projection neurons $\left(N_{\mathrm{SG}} \%\right)$ to projections targeting areas V1 (K89, neonatal) and V4 (B02, fetal) in the immature macaque cortex. a Immature $N_{\mathrm{SG}} \%$ in relation to the corresponding adult $N_{\mathrm{SG}} \%$. b $N_{\mathrm{SG}} \%$ for both immature and adult cortex in relation to architectonic differentiation measured as difference in architectonic type, where type difference $=$ type $_{\text {source area }}-$ type $_{\text {target area }}$. c $N_{\mathrm{SG}} \%$ for both immature and adult cortex in relation to architectonic differentiation measured as difference in neuron density, where neuron density difference $=$ density $_{\text {source area }}-$ density $_{\text {target area }}$. d Fraction of supragranular projection neurons observed in the immature cortex that remains in the adult cortex in relation to difference in architectonic type. e Fraction of supragranular projection neurons observed in the immature cortex that remains in the adult cortex in relation to difference in neuron density. In these data, the supragranular contribution declines with maturation. That is, in $(\mathbf{d}, \mathbf{e})$, the value of adult $N_{\mathrm{SG}} \%$ divided by immature $N_{\mathrm{SG}} \%$ is below 1 for most areas. Projection data from K89 (Kennedy et al. 1989) and B02 (Batardière et al. 2002) 

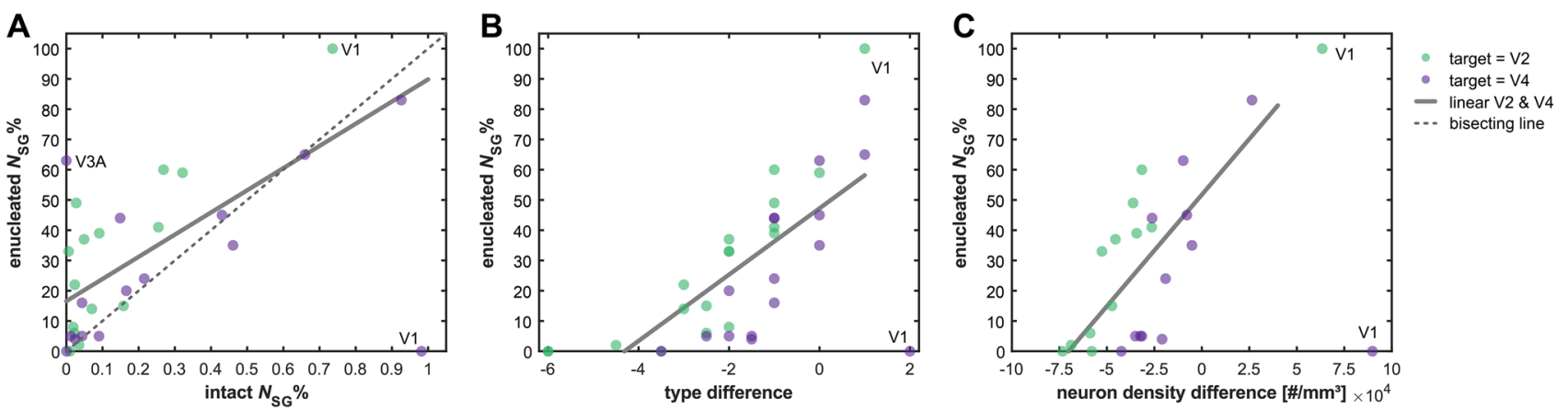

Fig. 2 Laminar projection patterns after enucleation. Relative contribution of supragranular projection neurons $\left(N_{\mathrm{SG}} \%\right)$ to projections targeting areas V2 and V4 in the cortex of enucleated macaque monkeys. $N_{\mathrm{SG}} \%$ after enucleation in relation to the respective $N_{\mathrm{SG}} \%$ in intact monkeys. b $N_{\mathrm{SG}} \%$ after enucleation in relation to architectonic differentiation measured as difference in architectonic type, where type difference $=$ type $_{\text {source area }}-$ type $_{\text {target area }}$ c $N_{\mathrm{SG}} \%$ after enucleation in relation to architectonic differentiation measured as

for injections in the neonatal to the infant cortex. In agreement with our analyses in the previous section, a general decline in supragranular contribution with maturation can be seen, which increases the correspondence between the $N_{\mathrm{SG}} \%$-values of intact adults and enucleated infants once they matured to adults. However, it should be kept in mind that these results may also be affected by the choice of injection sites in the analysed studies. Particularly, these sites were all in late-developing, well-differentiated cortical areas (V1, V2, V4); thus, according to the structural model, different trends, such as developmental increases of supragranular projection contributions, may be expected for injections in less well-differentiated areas.

Despite the drastic effects of enucleation on the organization of the primary visual cortex, the gradual changes in laminar projection patterns that have been reported to align with the relative architectonic differentiation of connected areas can also be observed in enucleated infant monkeys (Fig. 2b, c, Online Resource 4). The laminar patterns of projections are strongly correlated with the relative architectonic differentiation of two connected areas, both when it is measured as difference in architectonic type and as difference in neuron density. Thus, despite possible changes in projection patterns, the previously observed relation between laminar patterns and relative differentiation still holds even after complete loss of visual input. In line with the drastic changes to the organization of V1 (cf. Magrou et al. 2018), projections from V1 appear to be altered most strongly. While the correlation of supragranular contribution with architectonic type difference or neuron density becomes stronger if $\mathrm{V} 1$ data points are excluded, it is strong and significant even if they are included. This observation implies that the establishment of regular laminar projection patterns difference in neuron density, where neuron density difference $=$ density $_{\text {source area }}-$ density $_{\text {target area. Data from V2 and V4 were pooled }}$ for correlations and linear regression. Projections originating in V1 were excluded from the linear regression because V1 was affected very strongly by the enucleation and the resulting $N_{\mathrm{SG}} \%$-values are outliers. Projection data from Chaudhuri et al. (2015), Magrou et al. (2018)

is largely independent of normal sensory input, with the possible exception of the directly perturbed areas.

\section{Discussion}

Here, we report that the characteristic laminar patterns of cortical projection origins, which are known to be closely associated with the relative architectonic differentiation of cortical areas (e.g., Barbas 1986; Hilgetag et al. 2016), are already correlated with the later adult architectonic differentiation in immature macaque monkey brains. This observation was made consistently in intact fetal and neonate macaque monkeys as well as in enucleated infant macaque monkeys. Hence, it appears that the processes that determine the layers from which a projection originates occur early in development, and are relatively robust to severe changes such as the loss of sensory input. Since we find that laminar projection patterns are consistent with the architectonic type principle soon after their establishment, processes that occur later during ontogenesis may play a smaller role in the emergence of the architectonic type principle. This applies, for example, to processes such as pruning, activity-dependent remodelling or selective apoptosis in the different layers across the cortical gradient. These phenomena may serve to refine further the laminar patterns of projection origins, but do not appear to have a crucial role in determining an overall bias towards infra- or supra-granular origins.

These observations concerning immature laminar patterns can inform attempts on explaining how the architectonic type principle may arise during development. Since early, robust processes appear to be sufficient for its emergence, later 
processes might be omitted from a mechanistic explanation of the origin of the principle without losing much explanatory power. That is, a mechanism that takes into account only early processes but disregards later processes should still be able to generate laminar patterns that do not diverge substantially from empirical observations. We previously demonstrated in silico that spatio-temporal interactions in a forming cortical sheet can give rise to connectivity that is consistent with the architectonic type principle in terms of the existence of projections between brain areas (Beul et al. 2018). The fact that immature projection patterns are already consistent with the architectonic type principle, as presented here, implies that such spatio-temporal interactions may also be sufficient for generating the typically observed laminar patterns of projection origins. If the underlying neurogenetic processes can be captured adequately, this concept might also provide an in silico explanation for the formation of characteristic laminar projection origins (Beul and Hilgetag 2020).

Of course, even if laminar projection patterns are determined early in brain development, there remain other candidate mechanisms besides emergence from spatio-temporal interactions. For example, genetic specification is very likely to play some role in the establishment of laminar projection patterns. It has been shown that the eventual projection fate is often acquired even prior to neuronal precursor migration (Jensen and Killackey 1984; McConnell 1988; Polleux et al. 2001) and that initial establishment of connectivity is largely independent of synaptic activity (Verhage et al. 2000). Guidance molecules and their receptors are often expressed in a cell-type specific manner, with many guidance molecules having dual actions depending on the type of receptor to which they bind (Castellani and Bolz 1997; Bagnard et al. 1998; Castellani et al. 1998; Kolodkin and Tessier-Lavigne 2011; Seiradake et al. 2016; Morales and Kania 2017; Stoeckli 2017). These combinations of guidance molecules and receptors have been shown to strongly constrain local, intra-areal connectivity (Bolz and Castellani 1997; Castellani and Bolz 1997). The same principle may apply to longer-range, inter-areal connections. The expression of guidance molecules and receptors is mediated by transcription factors, whose spatially and temporally finetuned expression gives rise to distinct cell types with diverse morphological and connectional properties and distinct functions. For example, corticofugal projection identity is mediated by the transcription factors encoded by genes such as Fezf2 and Ctip2, for example, reviewed in Molyneaux et al. (2007) and Gaspard and Vanderhaeghen (2011). The effect of Feszf2 expression is not only permissive, but also causal, as forced expression of Fezf2 in progenitors destined for upper layers can induce these cells to atypically project to the pons (Chen et al. 2005). Another example of the genetic specification of a broad class of projection neurons are callosally projecting neurons, of which there are both upper and lower layer populations. Expression of different genes such as Satb2, Hspb3, and Lpl appears to generally specify callosal projection neurons (Alcamo et al. 2008; Molyneaux et al. 2009), while there are also genes specific to either upper or lower layer callosal projection neurons [e.g., Dkk3, Nectin-3 or Plexin-D1 (Molyneaux et al. 2009)]. Since there is evidence for the genetic specification of anatomical projection patterns at small (intrinsic, intra-areal circuits) and large (e.g., corticofugal versus callosal projections) spatial scales, projections at intermediate spatial scales, such as cortico-cortical inter-areal projections, are not likely to be an exception from this mode of connection organization. For example, it has been shown that, while the white matter of the spinal cord is generally permissive for cortical axon growth, innervation of sections of the spinal grey matter is specific and topographically correct (Stanfield and O'Leary 1985; O'Leary and Stanfield 1986; Kuang and Kalil 1994; Kuang et al. 1994).

To sum up, we draw two main conclusions from the results presented here (Fig. 3). First, we show that already in the prenatal and neonatal cortex, the laminar patterns of projection origins correlate with the architectonic differentiation observed in the adult cortex, and that these laminar patterns are not substantially altered by complete loss of visual input. Second, it appears that the initially present biases in laminar projections patterns are progressively strengthened by later developmental processes. During this sharpening of laminar specificity, the degree of change that projections undergo in their supragranular contribution varies concurrently with the relative architectonic differentiation of the connected areas (Fig. 3c). These findings have implications for the organization of structural connectivity, indicating that early neurogenetic processes are sufficient to establish the typical laminar projection patterns during brain development. Using computational simulations, we have demonstrated how the architectonic type principle can arise from spatio-temporal interactions of neuronal populations in the forming brain (Beul et al. 2018), and have recently extended the simulations to demonstrate the determination of laminar patterns through early neurogenetic processes (Beul and Hilgetag 2020). Our present findings on immature laminar patterns of projection origins strengthen the support for such a mechanistic explanation of how the architectonic type principle emerges during ontogenesis.

Supplementary Information The online version contains supplementary material available at https://doi.org/10.1007/s00429-021-02219-6. 
Fig. 3 Summary of developmental changes in laminar projection patterns. The architecture of connected cortical areas varies along a natural axis of cortical organization (Hilgetag et al. 2019), such that their relative differentiation may change from a negative to a positive value, as the source area of a projection becomes more differentiated than the target area. a The architectonic type principle describes how the proportions of upper and deep laminar origins of projection neurons vary along this gradient of relative differentiation. $\mathbf{b}$ The present findings show that, already in the immature brain, the contribution of supragranular neurons to a given projection is the stronger, the more differentiated the source area is relative to the target area (immature NSG\%) (cf. Fig. 1b, c). c This pattern becomes more pronounced as the initially formed projections are refined by later developmental processes (adult NSG\%). Specifically, we observed that this refinement appears proportional to the relative differentiation of connected areas. While the supragranular contribution to projections mainly decreases, the magnitude of this decrease changes concurrently with relative differentiation and eventually reverses into an increase of supragranular contribution. This results in the progressive strengthening of initially present biases in laminar projection patterns (cf. Fig. 1d, e)
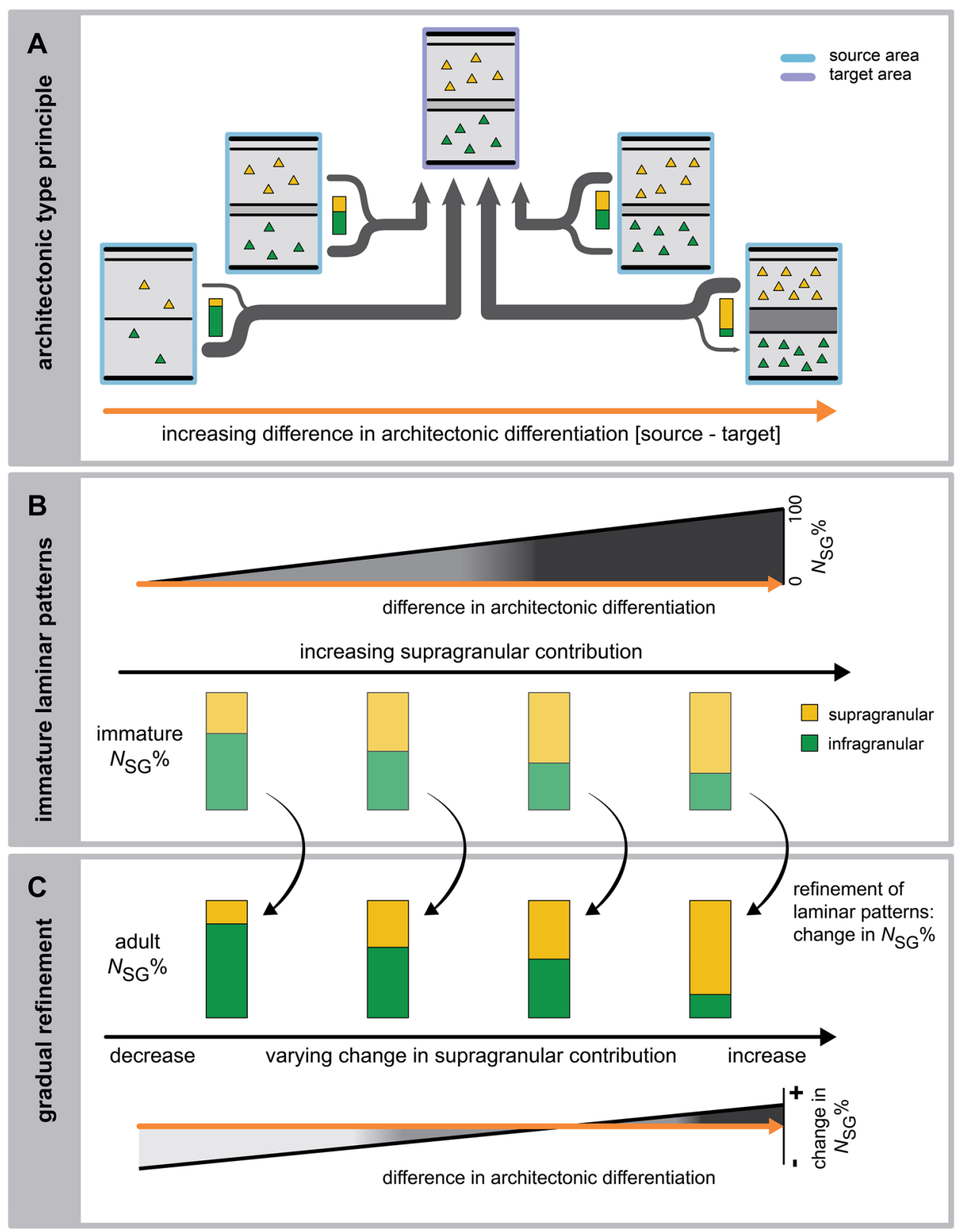

Acknowledgements This work was supported by grants of the German Research Council (DFG grants SFB 936/ A1, TRR 169/ A2, and SPP 2041) and the Human Brain Project (HBP/ SGA2, SGA3) to CCH.

Funding Open Access funding enabled and organized by Projekt DEAL. This study was supported by grants of the German Research Council (DFG grants SFB 936/ A1, TRR 169/ A2, and SPP 2041) and the Human Brain Project (HBP/ SGA2, SGA3) to CCH.

\section{Compliance with ethical standards}

Conflict of interest The authors declare that they have no conflict of interest.
Ethical approval This article does not contain any studies with human participants or animals performed by any of the authors.

Open Access This article is licensed under a Creative Commons Attribution 4.0 International License, which permits use, sharing, adaptation, distribution and reproduction in any medium or format, as long as you give appropriate credit to the original author(s) and the source, provide a link to the Creative Commons licence, and indicate if changes were made. The images or other third party material in this article are included in the article's Creative Commons licence, unless indicated otherwise in a credit line to the material. If material is not included in the article's Creative Commons licence and your intended use is not permitted by statutory regulation or exceeds the permitted use, you will need to obtain permission directly from the copyright holder. To view a copy of this licence, visit http://creativecommons.org/licenses/by/4.0/. 


\section{References}

Alcamo EA, Chirivella L, Dautzenberg M, Dobreva G, Fariñas I, Grosschedl R, McConnell SK (2008) Satb2 regulates callosal projection neuron identity in the developing cerebral cortex. Neuron 57:364-377

Bagnard D, Lohrum M, Uziel D, Püschel AW, Bolz J (1998) Semaphorins act as attractive and repulsive guidance signals during the development of cortical projections. Dev Camb Engl 125:5043-5053

Barbas H (1986) Pattern in the laminar origin of corticocortical connections. J Comp Neurol 252:415-422

Barbas H (2015) General cortical and special prefrontal connections: Principles from structure to function. Annu Rev Neurosci 38:269-289

Barbas H, Rempel-Clower NL (1997) Cortical structure predicts the pattern of corticocortical connections. Cereb Cortex 7:635-646

Barbas H, García-Cabezas MÁ (2016) How the prefrontal executive got its stripes. Curr Opin Neurobiol 40:125-134

Barbas H, Hilgetag CC, Saha S, Dermon CR, Suski JL (2005) Parallel organization of contralateral and ipsilateral prefrontal cortical projections in the rhesus monkey. BMC Neurosci 6:32

Barone P, Dehay C, Berland M, Bullier J, Kennedy H (1995) Developmental remodeling of primate visual cortical pathways. Cereb Cortex 5:22-38

Batardière A, Barone P, Knoblauch K, Giroud P, Berland M, Dumas A-M, Kennedy H (2002) Early specification of the hierarchical organization of visual cortical areas in the macaque monkey. Cereb Cortex 12:453-465

Beul SF, Hilgetag CC (2019) Neuron density fundamentally relates to architecture and connectivity of the primate cerebral cortex. NeuroImage 189:117051

Beul SF, Hilgetag CC (2020) Systematic modelling of the development of laminar projection origins in the cerebral cortex: Interactions of spatio-temporal patterns of neurogenesis and cellular heterogeneity. PLoS Comput Biol 16(10):e1007991

Beul SF, Grant S, Hilgetag CC (2015) A predictive model of the cat cortical connectome based on cytoarchitecture and distance. Brain Struct Funct 220:3167-3184

Beul SF, Barbas H, Hilgetag CC (2017) A predictive structural model of the primate connectome. Sci Rep 7:43176

Beul SF, Goulas A, Hilgetag CC (2018) Comprehensive computational modelling of the development of mammalian cortical connectivity underlying an architectonic type principle. PLoS Comput Biol 14:e1006550

Bolz J, Castellani V (1997) How do wiring molecules specify cortical connections? Cell Tissue Res 290:307-314

Castellani V, Bolz J (1997) Membrane-associated molecules regulate the formation of layer-specific cortical circuits. Proc Natl Acad Sci 94:7030-7035

Castellani V, Yue Y, Gao PP, Zhou R, Bolz J (1998) Dual action of a ligand for Eph receptor tyrosine kinases on specific populations of axons during the development of cortical circuits. J Neurosci 18:4663-4672

Chaudhuri R, Knoblauch K, Gariel M-A, Kennedy H, Wang X-J (2015) A large-scale circuit mechanism for hierarchical dynamical processing in the primate cortex. Neuron 88:419-431

Chen J-G, Rašin M-R, Kwan KY, Šestan N (2005) Zfp312 is required for subcortical axonal projections and dendritic morphology of deep-layer pyramidal neurons of the cerebral cortex. Proc Natl Acad Sci 102:17792-17797

Dombrowski SM, Hilgetag CC, Barbas H (2001) Quantitative architecture distinguishes prefrontal cortical systems in the rhesus monkey. Cereb Cortex 11:975-988
Feldman Barrett L, Simmons WK (2015) Interoceptive predictions in the brain. Nat Rev Neurosci 16:419-429

Felleman DJ, Van Essen DC (1991) Distributed hierarchical processing in the primate cerebral cortex. Cereb Cortex 1(1):1-47

Friston K (2010) The free-energy principle: a unified brain theory? Nat Rev Neurosci 11:127-138

García-Cabezas MÁ, Zikopoulos B, Barbas H (2019) The structural model: a theory linking connections, plasticity, pathology, development and evolution of the cerebral cortex. Brain Struct Funct 224(3):985-1008

Gaspard N, Vanderhaeghen P (2011) Laminar fate specification in the cerebral cortex. F1000 Biol Rep 3:6

Ghashghaei HT, Hilgetag CC, Barbas H (2007) Sequence of information processing for emotions based on the anatomic dialogue between prefrontal cortex and amygdala. NeuroImage 34:905-923

Goulas A, Uylings HBM, Hilgetag CC (2017) Principles of ipsilateral and contralateral cortico-cortical connectivity in the mouse. Brain Struct Funct 222:1281-1295

Goulas A, Betzel RF, Hilgetag CC (2019) Spatiotemporal ontogeny of brain wiring. Sci Adv 5(6):eaav9694

Hilgetag CC, Grant S (2010) Cytoarchitectural differences are a key determinant of laminar projection origins in the visual cortex. NeuroImage 51:1006-1017

Hilgetag CC, O'Neill MA, Young MP (2000) Hierarchical organization of macaque and cat cortical sensory systems explored with a novel network processor. Philos Trans R Soc Lond B Biol Sci 355(1393):71-89

Hilgetag CC, Medalla M, Beul SF, Barbas H (2016) The primate connectome in context: principles of connections of the cortical visual system. NeuroImage 134:685-702

Hilgetag CC, Beul SF, van Albada SJ, Goulas A (2019) An architectonic type principle integrates macroscopic cortico-cortical connections with intrinsic cortical circuits of the primate brain. Netw Neurosci 3(4):905-923

Jensen KF, Killackey HP (1984) Subcortical projections from ectopic neocortical neurons. Proc Natl Acad Sci 81:964-968

Karlen SJ, Krubitzer L (2009) Effects of bilateral enucleation on the size of visual and nonvisual areas of the brain. Cereb Cortex 19:1360

Kennedy H, Bullier J, Dehay C (1989) Transient projection from the superior temporal sulcus to area 17 in the newborn macaque monkey. Proc Natl Acad Sci 86:8093-8097

Kolodkin AL, Tessier-Lavigne M (2011) Mechanisms and molecules of neuronal wiring: A primer. Cold Spring Harb Perspect Biol 3:a001727

Kuang RZ, Kalil K (1994) Development of specificity in corticospinal connections by axon collaterals branching selectively into appropriate spinal targets. J Comp Neurol 344:270-282

Kuang RZ, Merline M, Kalil K (1994) Topographic specificity of corticospinal connections formed in explant coculture. Development 120:1937-1947

Magrou L, Barone P, Markov NT, Killackey HP, Giroud P, Berland M, Knoblauch K, Dehay C, Kennedy H (2018) How areal specification shapes the local and interareal circuits in a macaque model of congenital blindness. Cereb Cortex 28:3017-3034

Markov NT, Ercsey-Ravasz MM, Ribeiro Gomes AR, Lamy C, Magrou L, Vezoli J, Misery P, Falchier A, Quilodran R, Gariel MA, Sallet J, Gamanut R, Huissoud C, Clavagnier S, Giroud P, SappeyMarinier D, Barone P, Dehay C, Toroczkai Z, Knoblauch K, Van Essen DC, Kennedy H (2014) A weighted and directed interareal connectivity matrix for macaque cerebral cortex. Cereb Cortex 24:17-36

McConnell SK (1988) Fates of visual cortical neurons in the ferret after isochronic and heterochronic transplantation. J Neurosci 8:945-974 
Medalla M, Barbas H (2006) Diversity of laminar connections linking periarcuate and lateral intraparietal areas depends on cortical structure. Eur J Neurosci 23:161-179

Medalla M, Lera P, Feinberg M, Barbas H (2007) Specificity in inhibitory systems associated with prefrontal pathways to temporal cortex in primates. Cereb Cortex 17:i136-i150

Molyneaux BJ, Arlotta P, Menezes JRL, Macklis JD (2007) Neuronal subtype specification in the cerebral cortex. Nat Rev Neurosci 8:427-437

Molyneaux BJ, Arlotta P, Fame RM, MacDonald JL, MacQuarrie KL, Macklis JD (2009) Novel subtype-specific genes identify distinct subpopulations of callosal projection neurons. J Neurosci 29:12343-12354

Morales D, Kania A (2017) Cooperation and crosstalk in axon guidance cue integration: additivity, synergy, and fine-tuning in combinatorial signaling. Dev Neurobiol 77:891-904

O'Leary DD, Stanfield BB (1986) A transient pyramidal tract projection from the visual cortex in the hamster and its removal by selective collateral elimination. Brain Res 392:87-99

Polleux F, Dehay C, Goffinet A, Kennedy H (2001) Pre- and postmitotic events contribute to the progressive acquisition of area-specific connectional fate in the neocortex. Cereb Cortex 11:1027-1039

Rempel-Clower NL, Barbas H (2000) The laminar pattern of connections between prefrontal and anterior temporal cortices in the
Rhesus monkey is related to cortical structure and function. Cereb Cortex 10:851-865

Rockland KS, Pandya DN (1979) Laminar origins and terminations of cortical connections of the occipital lobe in the rhesus monkey. Brain Res 179:3-20

Seiradake E, Jones EY, Klein R (2016) Structural perspectives on axon guidance. Annu Rev Cell Dev Biol 32:577-608

Stanfield BB, O'Leary DDM (1985) The transient corticospinal projection from the occipital cortex during the postnatal development of the rat. J Comp Neurol 238:236-248

Stoeckli E (2017) Where does axon guidance lead us? F1000Research 6:78

Verhage M, Maia AS, Plomp JJ, Brussaard AB, Heeroma JH, Vermeer H, Toonen RF, Hammer RE, van Den TK, Berg MM, Geuze HJ, Südhof TC (2000) Synaptic assembly of the brain in the absence of neurotransmitter secretion. Science 287:864-869

Publisher's Note Springer Nature remains neutral with regard to jurisdictional claims in published maps and institutional affiliations. 